\title{
Immunological efficacy of glycoconjugates derived from Vibrio cholerae O1 serotype Ogawa detoxified LPS in mice
}

Correspondence

Ema Paulovičová

ema.paulovicova@savba.sk

Received 24 March 2010

Accepted 12 August 2010

\author{
Ema Paulovičová, Jana Korcová, Pavol Farkaš and Slavomír Bystrický
}

Slovak Academy of Sciences, Institute of Chemistry, Centre of Excellence GLYCOMED, Dubravska cesta 9, 84236 Bratislava, Slovakia

\begin{abstract}
This study focused on changes in selected parameters of humoral and cellular immunity following vaccination of mice with unique Vibrio cholerae LPS-protein-complexed conjugates. The $V$. cholerae detoxified LPS (dLPS)-derived antigenic structures O-specific polysaccharide (O-SP) and de-O-acylated LPS (DeOAc-LPS) were used to prepare glycoconjugates by linking both dLPSs to glucan, the immunomodulating matrix, and then to BSA carrier. Animals were given a primary vaccination and boosted at 2 -week intervals with a dose of $4.5 \mu \mathrm{g}$ saccharide antigen. The last boost was given either subcutaneously or intraperitoneally (i.p.) to compare the boosting effect and to optimize the effective immunization route. Both conjugates (O-SP-BSA and DeOAc-LPS-BSA) induced significant levels of antigen-specific lg isotypes, especially $\lg G$ and IgM. The i.p. booster route was more effective. A T helper 1 response was achieved only by immunization with O-SP-BSA conjugate administered i.p. Significant acceleration of phagocytic capacity and respiratory burst of neutrophils was demonstrated by both immunogenic formulations. Activation of T- and B-cell adaptive immunities was exhibited as specific changes in CD3 : CD19 and CD4 : CD8 ratios, B-cell low-affinity Fc $\gamma$ II and III receptor expression and induction of CD45R antigen.
\end{abstract}

\section{INTRODUCTION}

Vibrio cholerae is the causal agent of the diarrhoeal disease cholera. Conventional cholera vaccine formulations use live-attenuated or inactivated organisms, or their subcellular moieties. Formulations containing LPS or LPSderived subcellular components could provide an alternative to existing vaccines. The immunogenicity of LPS is ascribed predominantly to core moieties and O-somatic antigen, which are suitable components for subcellular vaccine development. Protein carrier-coupled polysaccharides represent an effective immunogen to recruit T-cell participation and generate $\mathrm{T}$ - and B-cell immunological memory accompanied by long-term protective immunity (Goldblatt, 2000; Mosley et al., 1968). Several saccharide components have been proposed as immunogenic formulas in developmental conjugate vaccines (Boutonnier et al., 2001; Gupta et al., 1998; Kossaczka et al., 2000). Synthetic O-specific polysaccharide (O-SP) and synthetic fragments of the O-SP of $V$. cholerae, conjugated to a protein carrier, have been shown to be protective in mice (Chernyak et al., 2002; Saksena et al., 2005, 2006). These conjugate cholera vaccine candidates were able to induce

Abbreviations: $\mathrm{ADH}$, adipic acid dihydrazide; DeOAc-LPS, de-Oacylated LPS; dLPS, detoxified LPS; i.p., intraperitoneally; O-SP, Ospecific polysaccharide; s.c., subcutaneously.
IgM and IgG humoral responses (Boutonnier et al., 2001; Gupta et al., 1992). Much cholera vaccine research is based on detoxified LPS (dLPS) (Adams \& Siebeling, 1984; Gupta et al., 1992; Paulovičová et al., 2006). Novel investigations of glycoconjugates prepared from acid-detoxified $V$. cholerae O1 LPS have also revealed the production of serum with vibriocidal activity (Grandjean et al., 2009). The efficacy of neutrophil phagocytosis also reflects the immunomodulatory effect of conjugate vaccine candidates (Jansen et al., 2001; Lefeber et al., 2003; Rodríguez et al., 2001). Successful conjugate vaccines should be potent stimulators of cell-mediated responses (Hirano et al., 2006; Salerno-Gonçalves \& Sztein, 2006). The roles of B and T lymphocytes (Guttormsen et al., 1999; Hirano et al., 2006; Zuercher et al., 2005), memory $\mathrm{T}$ cells, and memory and plasma B cells following vaccination with conjugate vaccines (Kelly et al., 2009; Wade, 2006; Zuercher et al., 2005) have been reported. However, little is known about the expression of $\mathrm{T}$ - and $\mathrm{B}$-cell differentiation and activation antigens in response to conjugate vaccine immunization.

The aim of this study was to establish the immunological efficacy of two novel glycoconjugates derived from $V$. cholerae O1 dLPS. A previous report by us described the chemical structure and complexity of the LPS-derived glycoconjugates (Machová et al., 2002), and subsequent 
studies demonstrated the immunological efficacy of subcellular preparations (Paulovičová et al., 2006, 2010). The current data in a murine model should provide novel information on immunomodulation strategies in anti- $V$. cholerae subcellular vaccine design.

\section{METHODS}

\section{Preparation of LPS}

Cultivation of bacteria. $V$. cholerae $\mathrm{O} 1$ serotype Ogawa El Tor cells were grown aerobically at $30{ }^{\circ} \mathrm{C}$ in a liquid medium containing $10 \mathrm{~g}$ $\mathrm{NaCl} 1^{-1}$ and $10 \mathrm{~g}$ bacterial peptone $1^{-1}(\mathrm{pH}$ 8.6). Cultivation was stopped by centrifugation $\left(245 \mathrm{~g}, 30 \mathrm{~min}, 4{ }^{\circ} \mathrm{C}\right)$. The bacterial cells were suspended in distilled water, centrifuged $\left(245 \mathrm{~g}, 30 \mathrm{~min}, 4{ }^{\circ} \mathrm{C}\right)$ and killed by the addition of phenol. The biomass was then determined.

Isolation of LPS. Cell wall-associated LPS from $V$. cholerae was isolated using hot phenol/water extraction, according to the method described by Westphal \& Jann (1965).

Detoxification of LPS and preparation of the glycolipid fraction (DeOAc-LPS). The LPS was treated with anhydrous hydrazine and the product was passed through a G-50 Sephadex column. The freeze-dried product of de-O-acylated LPS (DeOAc-LPS) contained $<10 \mathrm{EU} \mu \mathrm{g}^{-1}$ and $<1 \%$ protein or nucleic acid. A Limulus amoebocyte lysate test for endotoxin estimation was performed using an E-Toxate kit (Sigma), following the manufacturer's instructions.

Preparation of the O-SP fraction. LPS used for O-SP-BSA conjugate preparation was treated with $2 \%$ acetic acid (Hisatsune et al., 1985) at $100{ }^{\circ} \mathrm{C}$ (detoxification procedure by mild acid hydrolysis) and then centrifuged to remove lipid A. The supernatant was dialysed and passed through a Sephacryl S-200 column. The freeze-dried purified product $(\mathrm{O}-\mathrm{SP})$ contained $<5 \%$ proteins and nucleic acids as contaminants.

\section{Preparation of O-SP-BSA and DeOAC-LPS-BSA}

Functional modification of O-SP and DeOAc-LPS. To $11.5 \mathrm{mg} \mathrm{O}-$ $\mathrm{SP}$ in $1 \mathrm{ml}$ water, $10.3 \mathrm{mg}$ adipic acid dihydrazide (ADH) and $39.3 \mathrm{mg}$ 4-(4,6-dimethoxy-1,3,5-triazin-2-yl)-4-methylmorpholinium chloride were added. The reaction mixture was stirred at room temperature for $20 \mathrm{~h}$ and the resulting suspension was dialysed, deionized and freeze-dried, yielding $9.6 \mathrm{mg}$ product $(83 \%)$ designated O-SP-ADH. DeOAc-LPS-ADH was prepared in the same way.

Preparation of O-SP-ADH and DeOAc-LPS-ADH with glucan matrix. To $9.0 \mathrm{mg}$ hydrolysed derivative of oxidized glucan (OXG-L2-13) (Farkaš \& Bystrický, 2008) in $2 \mathrm{ml}$ acetate buffer ( $\mathrm{pH} 4.5$ ), $9.1 \mathrm{mg} \mathrm{O}-\mathrm{SP}-\mathrm{ADH}$ in $1 \mathrm{ml}$ acetate buffer was added. The reaction mixture was stirred at room temperature for $96 \mathrm{~h}$. $\mathrm{Na}\left(\mathrm{BH}_{3}\right) \mathrm{CN}$ (2 mg) was added, and the reaction mixture was stirred for another $3 \mathrm{~h}$ at room temperature and then sedimented by centrifugation. The supernatant was purified on a Sepharose CL6B column. Fractions were analysed by ${ }^{1} \mathrm{H}-\mathrm{NMR}$ spectroscopy. Freeze-drying of the relevant fractions yielded $4.2 \mathrm{mg}$ (23\%) O-SPADH-OXG product. The degree of substitution (DS) was $2.3 \%$, estimated from ${ }^{1} \mathrm{H}-\mathrm{NMR}$. The DeOAc-LPS-ADH-OXG product was obtained under the same conditions from the hydrolysed derivative of oxidized glucan OXG-L3-15 (Farkaš \& Bystrický, 2008). DeOAc-LPS-ADH-OXG with a DS of $6.3 \%$ and a yield of $42 \%$ was produced.
Conjugation of O-SP-ADH-OXG and DeOAc-LPS-ADH-OXG products to BSA. To $3.0 \mathrm{mg} \mathrm{O}-\mathrm{SP}-\mathrm{ADH}-\mathrm{OXG}$ conjugate dissolved in $1 \mathrm{ml}$ acetate buffer $\left(0.05 \mathrm{~mol} \mathrm{l}^{-1}, \mathrm{pH} \mathrm{5.0}\right)$ was added $1 \mathrm{mg}$ BSA in $0.5 \mathrm{ml}$ acetate buffer. The reaction mixture was stirred at room temperature for $18 \mathrm{~h} . \mathrm{NaBH}_{4}(5 \mathrm{mg})$ was added to quench the reaction. The solution was centrifuged and the supernatant was purified on a Sepharose CL-6B column. Fractions containing the conjugate were pooled and freeze-dried, giving a yield of $82 \%$. The product was designated O-SP-BSA conjugate with a $M_{\mathrm{r}}$ of 280000 , with a BSA content of $22 \%$ (estimated by the method of Bradford, 1976). The DeOAc-LPS-BSA conjugate was obtained under the same conditions, with a yield of $95 \%, M_{\mathrm{r}}$ of 330000 and containing $12 \%$ BSA.

Animals and immunization schedule. Female BALB/c mice aged 8-12 weeks were obtained from the breeding facility Velaz (Prague, Czech Republic). Animal experiments were conducted in compliance with the Organization for Economic Co-operation and Development Good Laboratory Practice guidelines, according to the ethical guidelines issued by the Research Base of Slovak Medical University, Institute of Preventive and Clinical Medicine (Bratislava, Slovakia). The primary and two booster immunizations of $4.5 \mu \mathrm{g} \mathrm{O}-$ SP-BSA containing $78 \%$ polysaccharide or $4.5 \mu \mathrm{g}$ DeOAc-LPS-BSA with $88.5 \%$ polysaccharide were administrated subcutaneously (s.c.) at 2-week intervals. Each experimental group comprised 10 animals. The immunization schedule also included a control (placebo) group of 10 mice that were injected with saline. After the first booster (second injection), the mice were split into two groups and the last booster (third injection) was administered either s.c. or intraperitoneally (i.p.) to compare the boosting effect and to optimize the most effective immunization route.

Blood specimens were obtained by retro-orbital puncture. Serum samples for antibody determination and lithium heparin and potassium EDTA blood samples for cytometric evaluation were collected 2 weeks after each immunization. Animals were killed by cervical dislocation. Pre-immune serum samples were collected as a negative-control reference before the primary vaccination.

Quantitative determination of anti-LPS immunoglobulin isotypes (IgG, IgM and IgA). ELISA plates (Immulon 4HBX microplates; Dynex) were coated overnight at $4{ }^{\circ} \mathrm{C}$ with $10 \mu \mathrm{g}$ LPS $\mathrm{ml}^{-1}$ in carbonate coating buffer ( $\mathrm{pH}$ 9.6). Following three washes with PBS and blocking with non-fat milk ( $2 \%$, w/v; KPL), serial dilutions of the tested sera were added to each well and incubated for $2 \mathrm{~h}$ at room temperature. Bound immunoglobulins were detected using alkaline phosphatase-conjugated anti-mouse IgG, IgA (both diluted $1: 3000)$ or IgM $(1: 1500)$, and Cell Lab goat anti-mouse IgG $(\mathrm{H}+\mathrm{L})$, goat anti-mouse IgA and goat anti-mouse IgM ( $\mu$-chain specific) (Beckman Coulter). The enzyme reaction was developed using a BluePhos Microwell phosphatase substrate system (KPL). The final absorbance was measured at $630 \mathrm{~nm}$. The concentrations of anti-LPS-specific isotypes are given as $\mathrm{ng}(\mathrm{ml} \text { serum })^{-1}$ and presented as the mean $\pm \mathrm{SD}$ of five mice per group. The exact quantification of

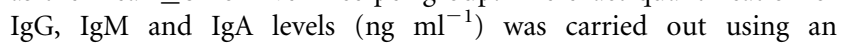
appropriate calibration curve based on reference mouse serum with known Ig levels (Mouse Reference Serum; Bethyl Laboratories).

Isotype and isotype subclass determination. Mouse sera IgA and IgG isotypes and subclasses IgG1, IgG2a, IgG2b and IgG3, including both $\kappa$ and $\lambda$ light chains, were examined using strip-immobilized goat anti-mouse antibodies (Serotec). Aliquots (150 $\mu$, diluted $1: 10000$ in PBS) of pooled pre-immune, conjugate and whole-cell sera were mixed with a colour detector complex (anti- $\kappa /$ anti- $\lambda$ antibodies coupled to coloured microparticles) and then allowed to react with equal amounts of strip-immobilized goat anti-mouse antibodies. The intensity of the resulting visual signal in a pattern of 
distinct bands was indicative of the specific antibody concentrations in the tested antisera and was evaluated via measured and calculated integral absorbance using UN-SCAN-IT gel software, version 1 (Silk Scientific).

Immunophenotyping. Whole peripheral blood was used for direct staining of cells and evaluation by flow cytometry using a Beckman Coulter FC 500 flow cytometer running under CXP software. For each sample, fluorescence histograms of 10000 cells were generated and analysed. The following rat anti-mouse phycoerythrin (PE)- and FITC-conjugated monoclonal antibodies were used: anti-CD3-FITC, anti-CD8-PE, anti-CD4-FITC, anti-CD25-FITC/PE, anti-CD19FITC, anti-CD45-PE, anti-MHC II-PE, anti-CD11b-FITC and antiCD16/32-PE (all from Antigenix America). The appropriate isotype antibody-negative controls (anti-IgG1 or anti-IgG2a) were used separately. The conjugated monoclonal antibodies $(5 \mu \mathrm{l})$ and wholeblood samples ( $50 \mu \mathrm{l}$ in lithium heparin) were added to $5 \mathrm{ml}$ sterile Falcon tubes (Becton Dickinson) and incubated for $30 \mathrm{~min}$ in the dark at $4{ }^{\circ} \mathrm{C}$. Lysis of erythrocytes was performed with $250 \mu$ l Optilyse $\mathrm{C}$ lysing solution (Immunotech). The lysing process was stopped after 30 min of incubation in the dark at $4{ }^{\circ} \mathrm{C}$ with PBS ( $\mathrm{pH} 7.2$ ). Finally, the samples were evaluated by a single- and/or dual-colour flow cytometric assay.

Immunocytometric evaluation of simultaneous phagocytosis and oxidative burst. Briefly, the double fluorescence of FITClabelled ingested Staphylococcus aureus cells and of hydroxyethidine (Polysciences) oxidized to ethidium bromide throughout phagocytosis accompanied by the respiratory burst of mouse granulocytes was evaluated by flow cytometry, as described above. For each blood sample, a fluorescence histogram of 5000 cells was generated and analysed. The gates were set around granulocytes to exclude debris. Measurements of phagocytosis (i.e. the ingestion of bacteria) took place under controlled conditions using FITClabelled opsonized S. aureus (SPA-FITC; Molecular Probes). Metabolic activity was determined by the oxidative burst of the stimulated transformation of non-fluorescent hydroxyethidine into fluorescent ethidium bromide following SPA-FITC ingestion. Aliquots of peripheral blood in lithium heparin were incubated with hydroxyethidine $(15.75 \mathrm{mg}$ in $5 \mathrm{ml}$ dimethylformamide; Merck) for $15 \mathrm{~min}$ at $37^{\circ} \mathrm{C}$. After treatment with SPA-FITC for a further $15 \mathrm{~min}$ at $37^{\circ} \mathrm{C}$, the reaction was stopped on ice. Subsequent erythrocyte lysis was performed for $15 \mathrm{~min}$ with an icecold ammonium chloride/potassium chloride lysis buffer $(200 \mathrm{ml}$ deionized water containing $1.658 \mathrm{~g} \mathrm{NH}_{4} \mathrm{Cl}, 0.2 \mathrm{~g} \mathrm{KHCO}_{3}, 7.4 \mathrm{mg}$ $\mathrm{Na}_{2}$ EDTA, pH 7.2-7.4).

The mean percentage of phagocytic cells represented the percentage of granulocytes that ingested at least one SPA-FITC from all granulocytes. The mean percentage of respiratory burst represented the percentage of granulocytes tagged by ethidium bromide from all granulocytes. The mean metabolic activity percentage represented the percentage of granulocytes that ingested at least one SPA-FITC and were tagged by ethidium bromide from all granulocytes.

Computational statistical analyses. Experimental results were calculated as means $\pm \mathrm{SD}$. The normality of distribution was evaluated according to the Shapiro-Wilk's test at the 0.05 level of significance. Statistical comparisons between experimental groups were performed using one-way analysis of variance (ANOVA) and post hoc Bonferroni and Tukey tests. The results were significant if the difference between the analysed groups equalled or exceeded the $95 \%$ confidence level $(P<0.05)$.

Statistics were performed with ORIGIN 7.5 PRO software (OriginLab). Pearson's correlation coefficient $(r)$ was used to compare the strength of the relationship between immunological parameters.

\section{RESULTS}

\section{Antibody-mediated immunity}

The levels of IgG, IgM and IgA against LPS were measured at day 0 and then 2 weeks after each immunization throughout the experiment. The antibody levels were compared with pre-immune serum levels and/or placebo. Comparisons of experimental groups were carried out at $1: 100$ antibody dilution.

Both conjugates elicited a statistically significant $\operatorname{IgG}$ antibody response after the first dose $(P<0.01)$ (Figs 1 and 2 ). However, only DeOAc-LPS-BSA elicited significant IgG levels after the booster applications (s.c. inoculation, $P<0.01$; i.p. inoculation, $P<0.05)$. The specific response after s.c. inoculation of O-SP-BSA was lower than after the second injection $(P<0.05)$, but when the third injection was applied by i.p. inoculation, it remained statistically significant $(P<0.01)$. Comparison of peak IgG levels of both conjugates after the third injection against reference levels indicated a significant increase. O-SP-BSA produced an increased antibody response that was 3.9 times higher after s.c. application and 5.4 times higher after i.p. application than the reference. In comparison with placebo, O-SP-BSA induced an 11.4 -fold increase $(P<0.01)$ in IgG response after s.c. inoculation and a 15.7 -fold increase $(P<0.001)$ after i.p. inoculation. DeOAc-LPS-BSA achieved a 5.9-fold
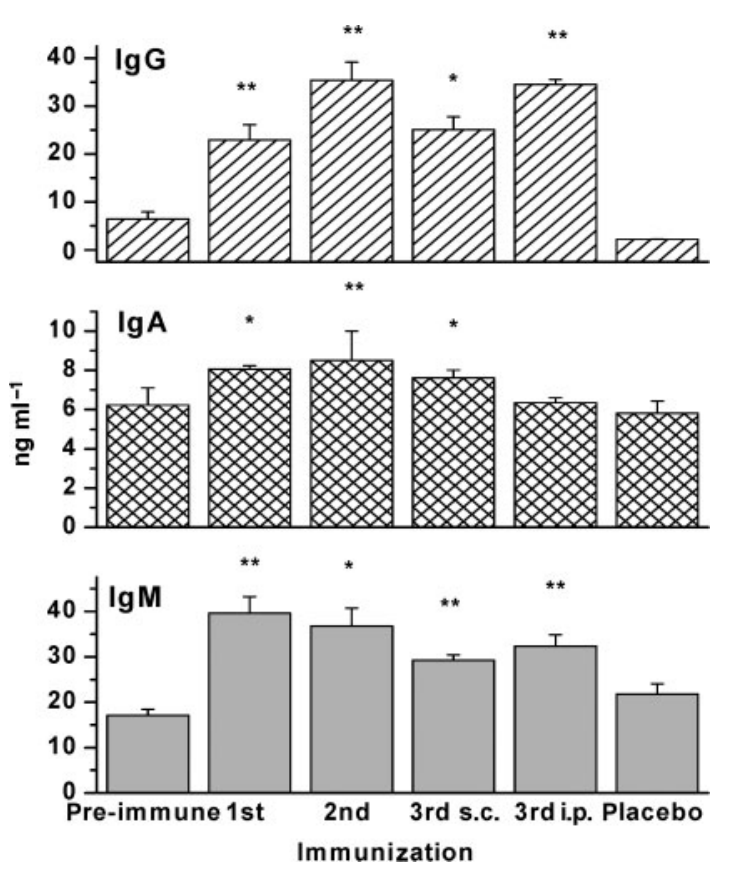

Fig. 1. Induction of Ig isotypic LPS-specific responses by O-SPBSA. Comparisons of all results from the vaccinated groups with the pre-immune reference serum results were performed using ANOVA. The experimental data are expressed as geometric means $\pm \mathrm{SD}$. ${ }^{* *}, P<0.01 ;{ }^{*}, P<0.05$. Differences were considered significant where $P<0.05$. 

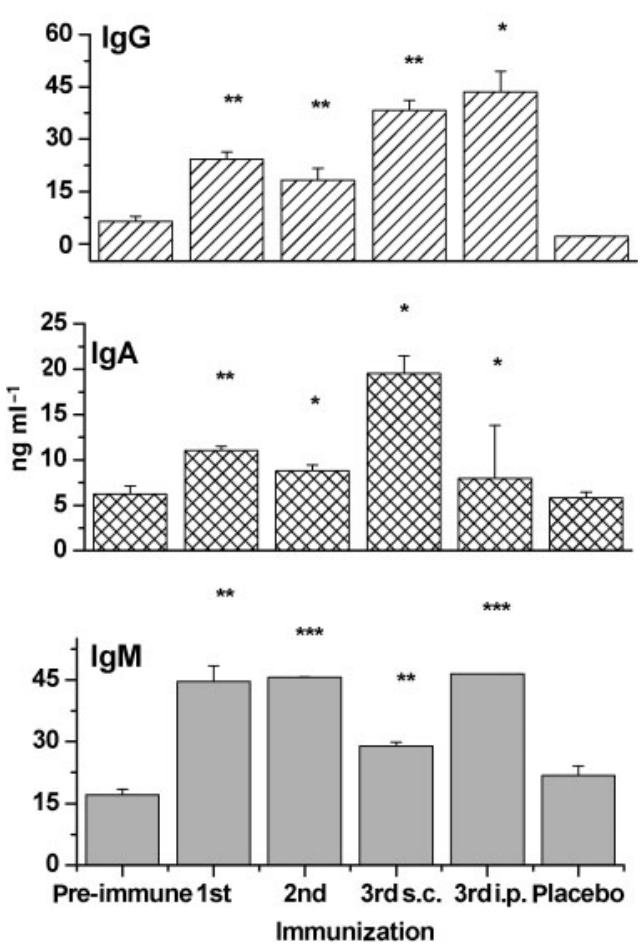

Fig. 2. Induction of Ig isotypic LPS-specific responses by DeOAcLPS-BSA. Comparisons of all results from the vaccinated groups with the pre-immune reference results were performed using ANOVA. The experimental data are expressed as geometric means \pm SD. ${ }^{* * *}, P<0.001 ;{ }^{* *}, P<0.01 ;{ }^{*}, P<0.05$. Differences were considered significant where $P<0.05$.

increase in antibody response after s.c. application and a 6.8fold increase after i.p. application compared with the preimmune baseline. DeOAc-LPS-BSA induced a 17.4-fold increase $(P<0.01)$ in antibody response after s.c. inoculation and a 19.7 -fold increase $(P<0.01)$ after i.p. inoculation compared with placebo. IgG anti-LPS titres of $1: 1600$ were found after the third immunization with both conjugates.

Detection of specific IgM anti-LPS conjugates resulted in a significant increase after the first two injections (O-SP-BSA $P<0.01$, and DeOAc-LPS-BSA $P<0.001$, after the second injection) (Figs 1 and 2). A slightly decreasing tendency was noted after the third injection of O-SP-BSA by s.c. and i.p. inoculation, although serum levels remained statistically significant $(P<0.01)$ compared with the reference levels. A decreasing tendency after the third injection of DeOAc-LPS-BSA was also noted with s.c. application. The increased antibody response after i.p. application was as statistically significant as after the second inoculation. OSP-BSA elicited slightly lower levels of IgM (32.4 $\mathrm{ng} \mathrm{ml}^{-1}$ ) than IgG $\left(34.5 \mathrm{ng} \mathrm{ml}^{-1}\right)$ after i.p. application, whilst DeOAc-LPS-BSA elicited lower or approximately the same levels of IgM (s.c., $28.9 \mathrm{ng} \mathrm{ml}^{-1}$; i.p., $46.4 \mathrm{ng} \mathrm{ml}^{-1}$ ) as IgG (s.c., $38.3 \mathrm{ng} \mathrm{ml}^{-1}$; i.p., $43.5 \mathrm{ng} \mathrm{ml}^{-1}$ ) after the third injection. The decreasing tendency of IgM serum levels after the third injection may indicate an $\operatorname{IgM}-\operatorname{IgG}$ isotype switch. The antibody titres were greater than $1: 1600$ after the third s.c. injection with O-SP-BSA and the third i.p. injection in the case of DeOAc-LPS-BSA. When compared with placebo, there were slightly statistically significant differences.

The O-SP-BSA conjugate elicited an IgA antibody response of statistically low significance $(P<0.05)$ throughout the experiment, with the maximal peak after the second injection (Fig. 1). The DeOAc-LPS-BSA conjugate elicited a statistically significant increase in anti-LPS IgA after the first injection $(P<0.01)$ (Fig. 2). An increasing tendency was also noted after the third injection compared with the reference (s.c. and i.p., $P<0.05$ ) and with placebo (s.c., $P<0.05$; i.p., $P<0.01)$. The increase in IgA antibody response was found to be 3.1-fold higher after the third s.c. injection in comparison with the reference and approximately the same in comparison with placebo. The antibody titres of IgA antibodies were not greater than $1: 200$ with the two conjugates.

\section{Cellular immunity}

Immunophenotyping and multiparameter flow cytometry revealed distinct immunization-associated changes in $\mathrm{T}$ and B-lymphocyte subsets and expression of activation antigens.

An increase in the number of B cells was observed after the first booster with both conjugates in comparison with placebo (O-SP-BSA, 1.36-fold increase; DeOAc-LPS-BSA, 1.49-fold increase). The kinetic trend in CD3: CD19 ratio confirmed this result (Fig. 3). An increase in B-cell counts after the second booster was not observed with either conjugate. Again, the kinetic trend in $\mathrm{CD} 3: \mathrm{CD} 19$ ratio confirmed this result (Fig. 3).

The tendency for expression of low-affinity Fc $\gamma$ II and III receptors (CD32 and CD16) resembled that of CD19 antigen (Fig. 4). Expression of $\mathrm{Fc} \gamma$ receptors differed not only with conjugate but also with immunization mode. For the O-SP-BSA conjugate applied by s.c. inoculation, expression was slightly higher (1.05 times) than with i.p. booster. Moreover, significantly higher expression in comparison with placebo also persisted after both secondbooster doses. In contrast, DeOAc-LPS-BSA was more effective when the boost was given by i.p. inoculation.

The impact of immunocompetency of both immunogens and different booster routes could also be seen on the ratio of CD3 : CD19 antigen (Fig. 3). DeOAc-LPS-BSA given i.p. induced predominantly CD3 expression over CD19, whilst the O-SP-BSA was more effective by the s.c. route.

Upregulation of $\mathrm{CD}^{+}$and $\mathrm{CD} 8^{+} \mathrm{T}$ cells by both immunogenic conjugates was also assessed (Fig. 5). The changes in T-cell expression of CD4 and CD8 surface antigens associated with active immunization were more significant with DeOAc-LPS-BSA than with O-SP-BSA, 


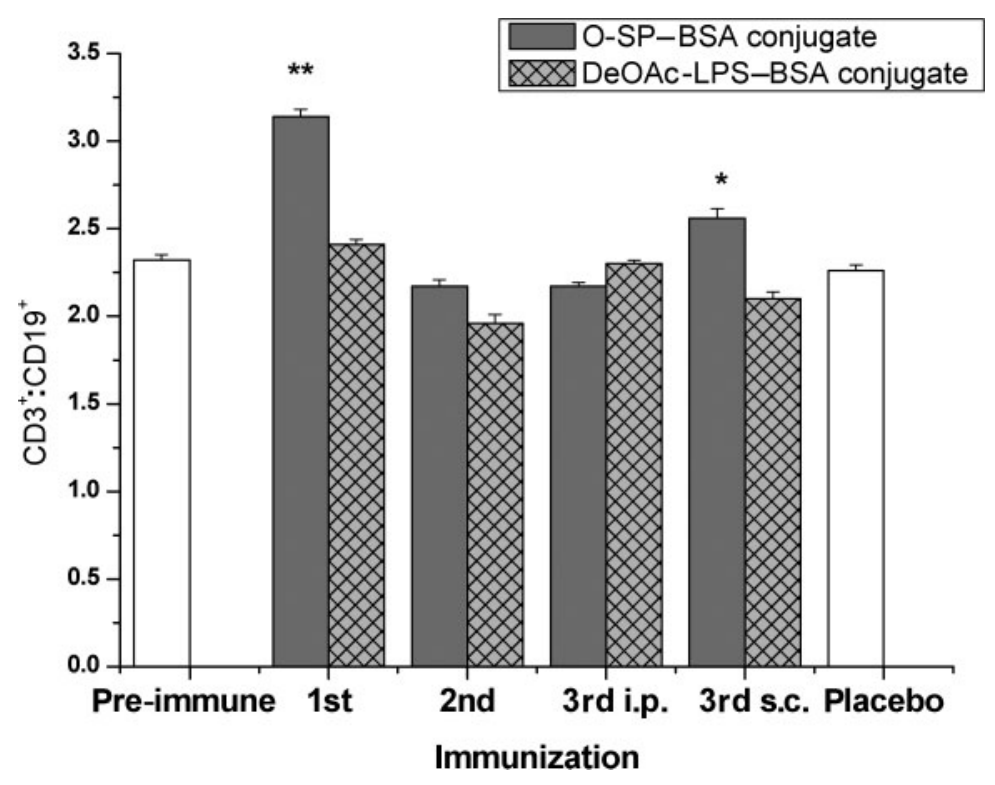

Fig. 3. Changes in CD3 : CD19 ratio throughout the experiment. The percentages of $\mathrm{CD}_{19}{ }^{+} \mathrm{B}$-cell and $\mathrm{CD}^{+}$T-cell populations were obtained from the 'lymphogate' $(n=10$, means $\pm S D$ ). Experimental data are expressed as geometric means $\pm \mathrm{SD}$. **, $P<0.01$; *, $P<0.05$. Differences were considered significant where $P<0.05$. especially after the primary vaccination (1.71-fold increase in $\mathrm{CD} 4$ : CD8 ratio vs placebo), whilst O-SP-BSA was more effective after both second boosters (1.5-fold increase in $\mathrm{CD} 4: \mathrm{CD} 8$ vs placebo).

Almost $99 \%$ expression of CD45 antigen on $\mathrm{CD} 19^{+}$cells was detected throughout the experiment, the increase representing $2.27 \%$ (DeOAc-LPS-BSA given i.p.) and $3.1 \%$ (O-SP-BSA given s.c.) versus placebo. Expression of $\mathrm{CD} 45 \mathrm{R}$ on $\mathrm{CD}^{+}$cells was accelerated by i.p. application of DeOAc-LPS-BSA ( $42 \%$ increase vs placebo) and O-SP-BSA (38\% increase vs placebo).

To determine the influence of both conjugates on $\mathrm{T}$ helper 1 (Th1)/Th2 polarization, Ig subclass isotyping was performed (Fig. 6). A steady increase in the $(\mathrm{IgG} 2 \mathrm{a}+\mathrm{IgG} 2 \mathrm{~b}): \operatorname{IgG} 1$ ratio, characteristic of a Th1 shift, could be seen only in the case of O-SP-BSA following the i.p. booster (3.26-fold increase vs pre-immune baseline). The expression of activation markers such as MHC II, $\mathrm{CD} 11 \mathrm{~b}$ or CD25 appeared not to be dependent on immunization by the two immunogens and the different immunization booster schemes (data not shown).

Simultaneous determination of phagocytosis and intracellular respiratory burst of neutrophils revealed the effective induction of innate immunity after the second booster with both immunogens. The O-SP-BSA conjugate statistically significantly stimulated phagocytic activity and respiratory burst via i.p. booster immunization $(P<0.001)$ in contrast
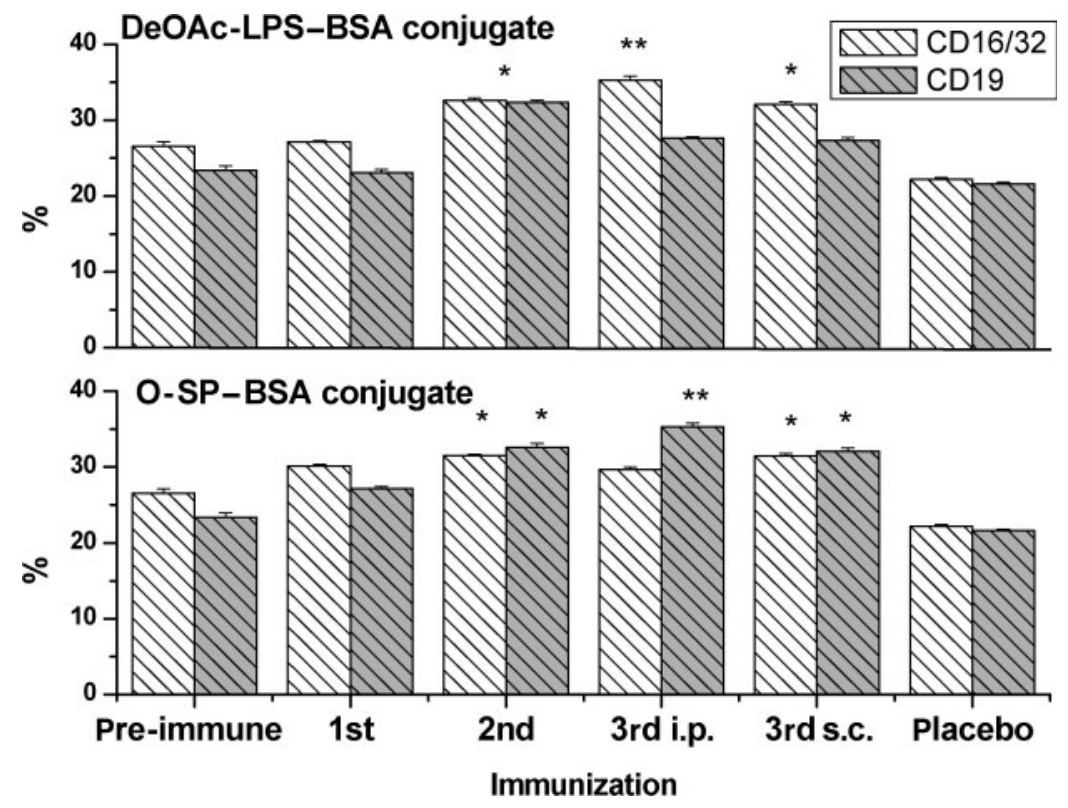

Fig. 4. Kinetic changes in expression of Fc $\gamma$ ॥ and III receptors and CD19 ${ }^{+}$cells during the immunization time schedule. The percentages of $\mathrm{CD}_{19}{ }^{+}$B-lymphocyte populations and CD16/32 ${ }^{+} \mathrm{FC} \gamma$ cells were obtained from the 'lymphogate' ( $n=10$, means $\pm \mathrm{SD})$. **,$P<0.01$; *, $P<0.05$. Differences were considered significant where $P<0.05$. 


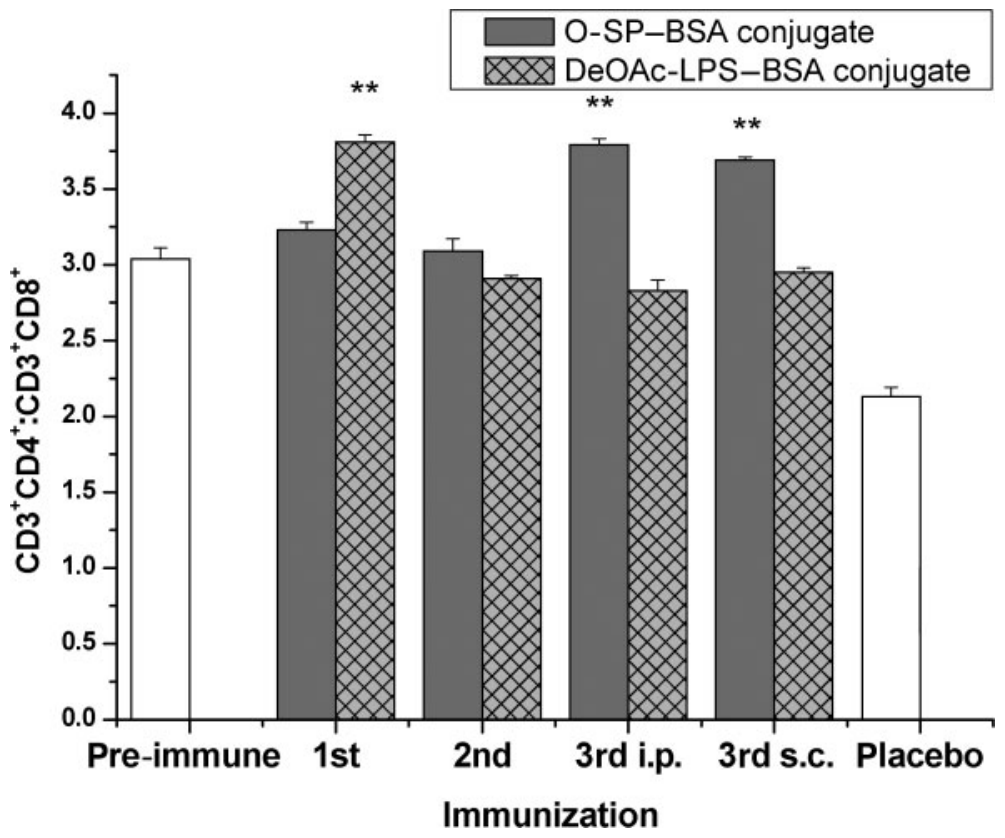

Fig. 5. $C D 4^{+}: C D 8^{+}$index of mouse peripheral blood on days 1,14 and 28 of immunization ( $n=10$, means $\pm \mathrm{SD})$. ${ }^{*}, P<0.01$. Differences were considered significant where $0.01<P<0.05$.

to the DeOAc-LPS-BSA conjugate, which stimulated phagocytic activity and respiratory burst via s.c. immunization $(P<0.001)$ (Fig. 7).

\section{DISCUSSION}

Parenterally administered killed whole-cell cholera O1 vaccine or orally administered attenuated cholera $\mathrm{O} 1$ strains elicit mainly IgM antibodies. In contrast, parenterally administered polysaccharide-protein conjugate vaccines elicit, in addition to IgM, high levels of serum anti-polysaccharide IgG. Induction of IgG is important due to its ability to penetrate the intestinal epithelium and initiate complement-mediated lysis of the bacterial inoculum (Kossaczka et al., 2000).

Novel conjugates constructed from dLPS of $V$. cholerae $\mathrm{O} 1$ Ogawa were synthesized under reaction conditions optimized to obtain a high degree of conjugation. Generally, the preparation of effective immunogenic conjugates is not easy because the size of the Ogawa O-SP is relatively small $(\sim 6000 \mathrm{Da})$. The immunogenicity of saccharides alone or in conjugates is directly related to their size (Gupta et al., 1992). Immunogenicity of the O-SP-BSA and DeOAc-LPSBSA conjugates was observed. Both conjugates elicited

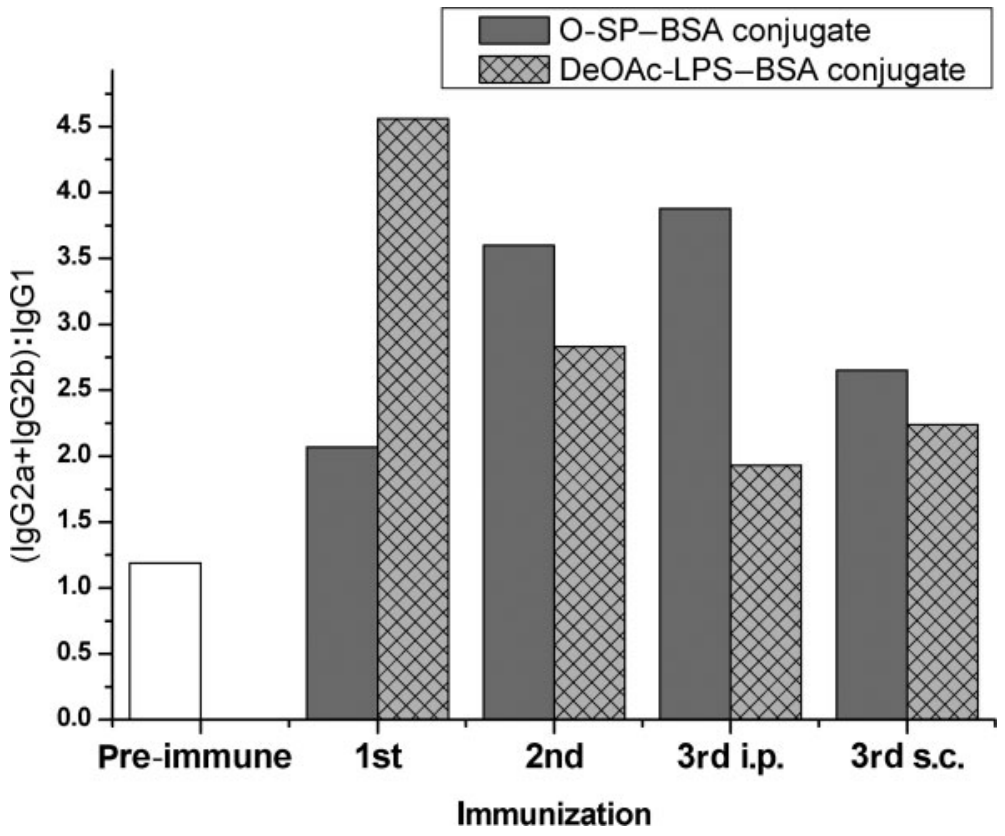

Fig. 6. Alterations in the $(\lg G 2 a+\lg G 2 b)$ : lgG1 ratio induced by the DeOAc-LPS-BSA and O-SP-BSA conjugates induced by the different immunization schemes. 


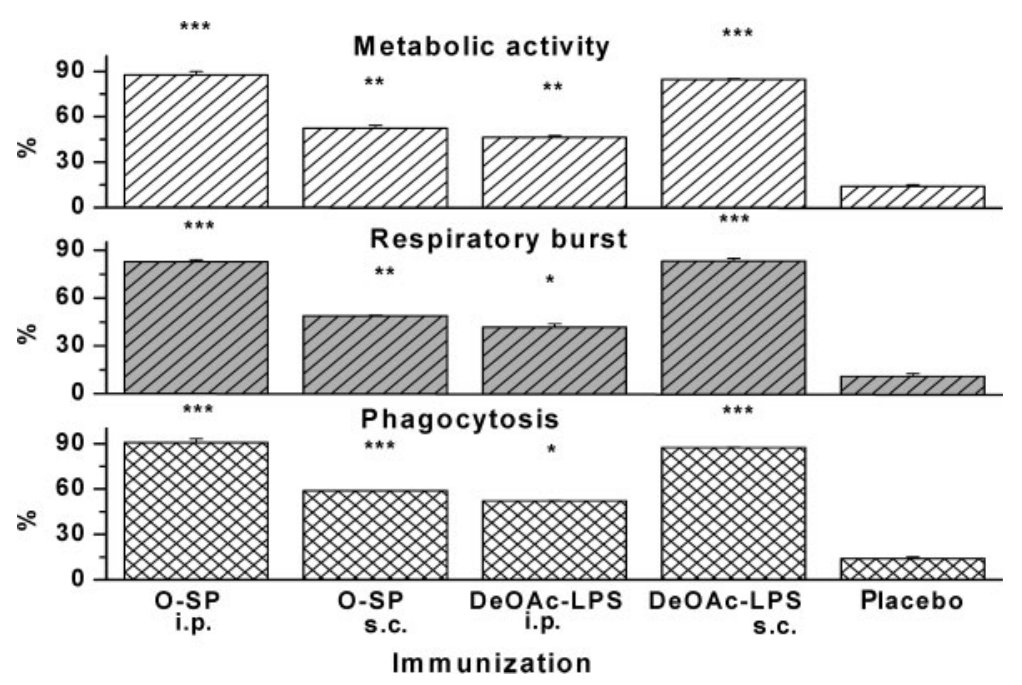

Fig. 7. Effect of immunization scheme and formulation used on simultaneous phagocytosis and intracellular respiratory burst of neutrophils. Comparisons of the second boosters of the O-SP-BSA and DeOAc-LPS-BSA conjugates with pre-immune levels were performed by ANOVA. The mean percentage of phagocytic cells represents the percentage of granulocytes that ingested at least one SPA-FITC compared with the total number of granulocytes. The mean percentage of respiratory burst represents the percentage of granulocytes tagged by ethidium compared with the total number of granulocytes. The mean metabolic activity percentage represents the percentage of granulocytes that ingested at least one SPA-FITC and were tagged by ethidium compared with the total number of granulocytes. The experimental data are expressed as geometric means \pm SD. ${ }^{* * *}, 0.000<P<0.001 ;{ }^{* *}, 0.001<P<0.01 ;{ }^{*}, 0.01<P<0.05$. Differences were considered significant where $0.01<P<0.05$.

significant amounts of antigen-specific IgG, IgM and IgA following the primary vaccination. The slightly different kinetics of IgG levels after immunization with O-SP-BSA in comparison with DeOAc-LPS-BSA could potentially have been due to: (i) the lower immunogenicity of the molecule (or a part of the molecule) as a result of simplicity (linear homopolymer of perosamine) (Gupta et al., 1992; Saksena et al., 2005), but both conjugates had the same length of Ospecific polysaccharide; (ii) the different chemical structures - the main part of DeOAc-LPS-BSA is a glycolipid containing a residue of lipid A from dLPS; (iii) the lipid structure possibly acting as an immunomodulator; or (iv) contamination by free polysaccharide. In practice, any free polysaccharide in the immunizing dose may suppress the development of anti-polysaccharide IgG-producing memory B cells. Suppression of a T-cell-dependent immune response has generally been observed for very high doses of free polysaccharide as a contaminant in polysaccharideprotein conjugate vaccines (Grandjean et al., 2009).

Multiple tests on cellular immunity by $\mathrm{T}$ - and B-cell immunophenotyping, activation antigen expression or phagocytosing capacity and metabolic burst revealed differences between the conjugates. $\mathrm{CD}_{1}{ }^{+}$cells are required for antibody production in response to vaccination. Thus, their proportion in peripheral blood circulation may serve as a correlate for a sufficiently induced humoral response by vaccination. To establish overall the cellimmunity response, the proportional representation of $\mathrm{CD}^{+}{ }^{+}$and $\mathrm{CD}_{1} 9^{+}$lymphocytes and $\mathrm{CD}^{+}$and $\mathrm{CD} 8{ }^{+}$
T-cell subpopulations (percentage of total lymphocytes or $\mathrm{CD}^{+} \mathrm{T}$ cells, respectively) was determined. The impact of dose administration and the different characteristics of the two conjugates could be seen. The B-/T-cell shift was more pronounced with O-SP-BSA administered i.p., in contrast to DeOAc-LPS-BSA where the s.c. route was more effective. Obviously, the characteristics of the immunogen, detoxification mode, attachment site and polysaccharideprotein ratio are important key parameters of induction of immunogenicity (Egan et al., 1995). The administration of LPS to mice not only activates all B cells but also triggers, as measured by the expression of lymphocyte activation markers, up to $80 \%$ of $\mathrm{CD}^{+}$and $\mathrm{CD}^{+}$T cells (Castro et al., 1998). In our study, the steady CD4:CD8 ratio increase was attributed to the O-SP-BSA conjugate, with the highest ratio being reached after the last booster and being accompanied by an increase in $\mathrm{CD}^{+}{ }^{+} \mathrm{CD} 4{ }^{+} \mathrm{T}$ cells. The observed changes in the expression of the activation marker $\mathrm{CD}_{2} 5^{+}$on $\mathrm{CD}^{+}{ }^{+}$and $\mathrm{CD} 8{ }^{+}$lymphocytes were without significant relevance. The expression of CD25 antigen on $\mathrm{T}$ cells occurs within $2-24 \mathrm{~h}$ and persists for only a few days after diminution of the stimulating antigen (Poulton et al., 1988). Activation of mouse B cells after LPS injection has been observed (Castro et al., 1998). The lowaffinity Fc $\gamma$ II and III receptors CD32 and CD16 expressed on normal murine pro-/pre-B cells are involved in the growth and differentiation of developing B-lineage cells (de Andres et al., 1998, 1999; Palermo et al., 1997). Immunization-induced expression of CD19 antigen correlates strongly with CD16/CD32 expression, and was more 
pronounced after vaccination with O-SP-BSA $(r=0.953$ vs $r=0.815$ for DeOAc-LPS-BSA). For O-SP-BSA, the s.c. route of booster administration was accompanied by higher levels of expression of CD19 and CD16/CD32; the CD3 : CD19 ratio also stressed this pattern. The activation of $\mathrm{B}$ cells by i.p. boost was obviously lower, as documented by $\mathrm{CD} 19^{+} \mathrm{CD} 16 / \mathrm{CD} 32^{+}$cells and the $\mathrm{CD} 3: \mathrm{CD} 19$ ratio. However, DeOAc-LPS-BSA induction of B cells and CD16/ CD32 expression by i.p. booster was greater than that induced by the s.c. booster; this finding was in agreement with the serum levels of IgG- and IgM-specific isotypes. Such B-cell involvement was also documented by a lower CD3 : CD19 ratio in comparison with s.c. immunization.

Human class II histocompatibility antigen (HLA-DR) is present on antigen-presenting cells, B cells, monocytes, thymic epithelial cells and macrophages. It is also expressed on activated $\mathrm{T}$ cells, and resting $\mathrm{T}$ cells can be induced to express HLA-DR antigen. It is of interest that crossreactions between mouse Ia and human HLA-D/DR antigens have been reported (Pierres et al., 1981). Based on HLA-DR accelerated expression after s.c. and i.p. administration of DeOAc-LPS-BSA (48 and 33\% increase vs pre-immune levels), it was concluded that antigen presentation and activation had been successful. The pattern of lymphocyte presentation and activation evaluated for expression of the leukocyte common antigen CD45R allowed complementary information on the characteristics of the novel glycoconjugates. CD45R is a $220 \mathrm{kDa}$ cell-surface molecule found predominantly on cells of B-cell lineage (pro-B to mature B cells), although it can be expressed by some activated T cells (Watanabe \& Akaike, 1994). Almost $99 \%$ expression on $\mathrm{CD} 19^{+}$cells was detected throughout the experiment, with the increase representing $2.27 \%$ (DeOAc-LPS given i.p.) and 3.1\% (OSP-BSA given s.c.) compared with placebo. The expression of $\mathrm{CD} 45 \mathrm{R}$ on $\mathrm{CD}^{+}$cells was accelerated by i.p. application of DeOAc-LPS-BSA ( $42 \%$ increase vs placebo) and O-SP-BSA ( $38 \%$ increase vs placebo). Th1 and Th2 cell involvement established a pro-Th1 pattern only in the case of O-SP-BSA booster administered i.p., accompanied by a steady increase in IgG2:IgG1 ratio throughout the experiment. T-cell dependency was also apparent on the basis of IgM/IgG shift (Figs 1 and 2) and CD3:CD19 ratio. The observed follow-up of IgG2: IgG1 ratio throughout the experiment suggested that, in the first phase of the immune response, both Th1 and Th2 responses were present, but that boosting vaccination seemed to be crucial. Conway et al. (2001) demonstrated that immunization by the i.p. route favours the induction of Thl cells, whereas the s.c. route favours Th2 cells. In addition to the adaptive branch of immune responses on vaccination, innate immunity represents an important vaccination target. Phagocytes represent the first line of defence in host innate immunity, as they are capable of phagocytosis and subsequent killing of microbial invaders, involving the production of reactive oxygen intermediates during oxidative burst (Gordon, 2007; Stuart \& Ezekowitz, 2005). The importance of phagocytosis and the antigen specificity of the opsonophagocytic response after vaccination has been stressed (Jansen et al., 2001; Lefeber et al., 2003; Rodríguez et al., 2001). Quantification of phagocytosis and oxidative burst enables us to study the impact of novel glycoconjugates on the functionality of neutrophils. Evidently, the different immunobiological properties of the novel glycoconjugates and the immunization routes influenced the metabolic and phagocytic activities of the neutrophils. The most effective phagocytosis accompanied by oxidative burst was observed after s.c. administration of the DeOAc-LPS-BSA conjugate and the i.p. booster with the O-SP-BSA conjugate (Fig. 7).

Our experimental data have shown several major points. They reveal a role for dLPS-derived novel immunogenic conjugates as inducers of both branches of immunity, adaptive and innate. Improved immunogenicity of protein-glucan matrix vaccine constructs is related to the effective activation of neutrophils as well as B- and T-cell populations during the vaccination. These data confirm that the process of detoxification did not influence the immunogenicity of the glycolipid and O-SP conjugates. This observation is particularly valuable for the future design of subcellular vaccine formulas derived from dLPS. The results stress the mutual relationships between structure and immunobiological behaviour.

In conclusion, it appeared that constructed conjugate based on O-SP derived from dLPS seems to be more suitable as a subcellular anti-cholera vaccine design than the glycolipidic moiety.

\section{ACKNOWLEDGEMENTS}

This work was supported by the Slovak Research and Development Agency under contract no. APVV-0032-06, by the Grant Agency of Slovak Academy of Sciences VEGA no. 2/7029/27 and by the Centre of Excellence GLYCOMED, CE SAS GLYCOMED.

\section{REFERENCES}

Adams, L. B. \& Siebeling, R. J. (1984). Production of Vibrio cholerae $\mathrm{O} 1$ and non-O1 typing sera in rabbits immunized with polysaccharide-protein carrier conjugates. J Clin Microbiol 19, 181-186.

Boutonnier, A., Villeneuve, S., Nato, F., Dassy, B. \& Fournier, J.-M. (2001). Preparation, immunogenicity, and protective efficacy, in a murine model, of a conjugate vaccine composed of the polysaccharide moiety of the lipopolysaccharide of Vibrio cholerae $\mathrm{O} 139$ bound to tetanus toxoid. Infect Immun 69, 3488-3493.

Bradford, M. M. (1976). A rapid and sensitive method for the quantitation of microgram quantities of protein utilizing the principle of protein-dye binding. Anal Biochem 72, 248-254.

Castro, A., Bemer, V., Nóbrega, A., Coutinho, A. \& Truffa-Bachi, P. (1998). Administration to mouse of endotoxin from Gram-negative bacteria leads to activation and apoptosis of T lymphocytes. Eur $\mathrm{J}$ Immunol 28, 488-495.

Chernyak, A., Kondo, S., Wade, T. K., Meeks, M. D., Alzari, P. M., Fournier, J. M., Taylor, R. K., Kovac, P. \& Wade, W. F. (2002). Induction of protective immunity by synthetic Vibrio cholerae hexasaccharide derived from $V$. cholerae O1 Ogawa lipopolysaccharide bound to a protein carrier. J Infect Dis 185, 950-962. 
Conway, M. A., Madrigal-Estebas, L., McClean, S., Brayden, D. J. \& Mills, K. H. G. (2001). Protection against Bordetella pertussis infection following parenteral or oral immunization with antigens entrapped in biodegradable particles: effect of formulation and route of immunization on induction of Th1 and Th2 cells. Vaccine 19, 1940-1950.

de Andres, B., Mueller, A. L., Verbeek, S., Sandor, M. \& Lynch, R. G. (1998). A regulatory role for $F c \gamma$ receptors CD16 and CD32 in the development of murine B cells. Blood 92, 2823-2829.

de Andres, B., Hagen, M., Sandor, M., Verbeek, S., Rokhlin, O. \& Lynch, R. G. (1999). A regulatory role for $\mathrm{Fc} \gamma$ receptors (CD16 and 32) in hematopoiesis. Immunol Lett 68, 109-113.

Egan, W., Frasch, C. E. \& Antony, B. F. (1995). Lot-release criteria, post licensure quality control and the Haemophilus influenzae type $\mathrm{B}$ conjugate vaccines. JAMA 273, 888-889.

Farkaš, P. \& Bystrický, S. (2008). Hydrolysis of the terminal dimethylacetal moiety on the spacers bound to carboxy groups containing glucans. Carbohydr Polym 74, 133-136.

Goldblatt, D. (2000). Conjugate vaccines. Clin Exp Immunol 119, 1-3. Gordon, S. (2007). The macrophage - past, present and future. Eur J Immunol 37, S9-S17.

Grandjean, C., Boutonnier, A., Dassay, B., Fournier, J.-M. \& Mulard, L. A. (2009). Investigation towards bivalent chemically defined glycoconjugate immunogens prepared from acid-detoxified lipopolysaccharide of Vibrio cholerae O1, serotype Inaba. Glycoconj J 26, 41-55.

Gupta, R. K., Szu, S. C., Filkenstein, R. A. \& Robbins, J. B. (1992). Synthesis, characterization, and some immunological properties of conjugates composed of the detoxified lipopolysaccharide of Vibrio cholerae $\mathrm{O} 1$ serotype Inaba bound to cholera toxin. Infect Immun 60, 3201-3208.

Gupta, R. K., Taylor, D. N., Bryla, D. A., Robbins, J. B. \& Szu, S. C. (1998). Phase 1 evaluation of Vibrio cholerae O1, serotype Inaba, polysaccharide-cholera toxin conjugates in adult volunteers. Infect Immun 66, 3095-3099.

Guttormsen, H. K., Sharpe, A. H., Chandraker, A. K., Brigtsen, A. K., Sayegh, M. H. \& Kasper, L. (1999). Cognate stimulatory B-cell-T-cell interactions are critical for $\mathrm{T}$-cell help recruited by glycoconjugate vaccines. Infect Immun 67, 6375-6384.

Hirano, T., Jiao, X., Chen, Z., Waes, C. V. \& Gu, X. X. (2006). Kinetics of mouse antibody and lymphocyte responses during intranasal vaccination with a lipooligosaccharide-based conjugate vaccine. Immunol Lett 107, 131-139.

Hisatsune, K., Yamamoto, F. \& Kondo, S. (1985). Lipopolysaccharide of Vibrio cholerae: chemical and serological properties. In Advances in Research on Cholera and Related Diarrhoeas, pp. 17-24. Edited by S. Kuwahara \& N. F. Pierce. Tokyo: KTK Scientific Publishers.

Jansen, W. T. M., Väkeväinen-Anttila, M., Käyhty, H., Nahm, M., Bakker, N., Verhoef, J., Snippe, H. \& Verheul, A. F. M. (2001). Comparison of a classical phagocytosis assay and a flow cytometry assay for assessment of the phagocytic capacity of sera from adults vaccinated with a pneumococcal conjugate vaccine. Clin Diagn Lab Immunol 8, 245-250.

Kelly, D. F., Snape, M. D., Perrett, K. P., Clutterbuck, E. A., Lewis, S., Blanchard-Rohner, G., Jones, M., Yu, L.-M. \& Pollard, A. J. (2009). Plasma and memory B-cell kinetics in infants following a primary schedule of $\mathrm{CRM}_{197}$-conjugated serogroup $\mathrm{C}$ meningococcal polysaccharide vaccine. Immunology 127, 134-143.

Kossaczka, Z., Shiloh, J., Johnsson, V., Taylor, D. N., Finkelstein, R. A., Robbins, J. B. \& Szu, S. C. (2000). Vibrio cholerae O139 conjugate vaccines: synthesis and immunogenicity of Vibrio cholerae O139 capsular polysaccharide conjugates with recombinant diphtheria toxin mutant in mice. Infect Immun 68, 5037-5043.
Lefeber, D. J., Benaissa-Trouw, B., Vliegenthart, J. F. G., Kamerling, J. P., Jansen, W. T. M., Kraaijeveld, K. \& Snippe, H. (2003). Th1directing adjuvants increase the immunogenicity of oligosaccharideprotein conjugate vaccines related to Streptococcus pneumoniae type 3. Infect Immun 71, 6915-6920.

Machová, E., Bystrický, S., Gáliková, A. \& Kogan, G. (2002). Preparation of a subcellular conjugate with the lipopolysaccharide from Vibrio cholerae 01 using $\beta$-D-glucan as matrix. Eur J Med Chem 37, 681-687.

Mosley, W. H., Feely, J. C. \& Pittman, M. (1968). The interrelationships of serological responses in humans, and the active mouse protection test to cholera vaccine effectiveness. In International Symposium on Enterobacterial Vaccines. Series on Immunobiological Standards 1968, vol. 15, pp. 185-196. Edited by R. H. Regamey, M. Stanic \& J. Ungar. Basel: Karger.

Palermo, M. S., Rosa, F. A., Alonso, G. F. \& Isturiz, A. (1997). Fc- $\gamma$ receptor-dependent clearance is enhanced following lipopolysaccharide in vivo treatment. Immunology 92, 536-543.

Paulovičová, E., Machová, E., Hoštacká, A. \& Bystrický, S. (2006). Immunological properties of complex conjugates based on Vibrio cholerae O1 Ogawa lipopolysaccharide antigen. Clin Exp Immunol 144, 521-527.

Paulovičová, E., Kováčová, E. \& Bystrický, S. (2010). Vibrio cholerae O1 Ogawa detoxified lipopolysaccharide structures as inducers of cytokines and oxidative species in macrophages. J Med Microbiol 59, 158-164.

Pierres, M., Rebouah, J. P., Kourilsky, F. M., Dosseto, M., Mercier, P., Mawas, C. \& Malissen, B. (1981). Cross-reactions between mouse Ia and human HLA-D/DR antigens analyzed with monoclonal alloantibodies. J Immunol 126, 2424-2429.

Poulton, T. A., Gallagher, A., Potts, R. C. \& Beck, J. S. (1988). Changes in activation markers and cell membrane receptors on human peripheral blood $\mathrm{T}$ lymphocytes during cell cycle progression after PHA stimulation. Immunology 64, 419-425.

Rodríguez, M. E., Van der Pol, W. L. \& Van de Winkel, J. G. J. (2001). Flow cytometry-based phagocytosis assay for sensitive detection of opsonic activity of pneumococcal capsular polysaccharide antibodies in human sera. J Immunol Methods 252, 33-44.

Saksena, R., Ma, X., Wade, T. K., Kováč, P. \& Wade, W. F. (2005). Effect of saccharide length on the immunogenicity of neoglycoconjugates from synthetic fragments of the O-SP of Vibrio cholerae O1, serotype Ogawa. Carbohydr Res 340, 2256-2269.

Saksena, R., Ma, X., Wade, T. K., Kováč, P. \& Wade, W. F. (2006). Length of the linker and the interval between immunizations influences the efficacy of Vibrio cholerae O1, Ogawa hexasaccharide neoglycoconjugates. FEMS Immunol Med Microbiol 47, 116-128.

Salerno-Gonçalves, R. \& Sztein, M. B. (2006). Cell-mediated immunity and the challenges for vaccine development. Trends Microbiol 14, 536-542.

Stuart, L. M. \& Ezekowitz, R. A. B. (2005). Phagocytosis: elegant complexity. Immunity 22, 539-550.

Wade, W. F. (2006). B-cell responses to lipopolysaccharide epitopes: who sees what first - does it matter? Am J Reprod Immunol 56, 329-336.

Watanabe, Y. \& Akaike, T. (1994). Activation signal induces the expression of B cell-specific CD45R epitope (6B2) on murine T cells. Scand J Immunol 39, 419-425.

Westphal, O. \& Jann, K. (1965). Bacterial lipopolysaccharides: extraction with phenol-water and further applications of the procedure. Methods Carbohydr Chem 5, 83-91.

Zuercher, A. W., Imboden, M. A., Jampen, S., Bosse, D., Ulrich, M., Chtioui, H., Lauterburg, B. H. \& Lang, A. B. (2005). Cellular immunity in healthy volunteers treated with an octavalent conjugate Pseudomonas aeruginosa vaccine. Clin Exp Immunol 142, 381-387. 